\title{
Tooth infection during the chemotherapy for malignant
}

\section{musculoskeletal tumor}

Yuka YAMADA ${ }^{1}$, Seiji ASODA ${ }^{1}$, Robert NAKAYAMA ${ }^{2}$, Terumi TAKEUCHI ${ }^{1}$, Yoko KUDO ${ }^{1}$, Hiromasa KAWANA ${ }^{3}$,Taneaki NAKAGAWA

1; Department of Dentistry and Oral Surgery, Keio University School of Medicine, 2; Department of Orthopaedic Surgery,

Keio University School of Medicine, 3; Department of Oral and Maxillofacial Implantology, Kanagawa Dental University,

\section{Introduction}

Tooth infection is often seen in the myelosuppression period after cytotoxic chemotherapy and can impede regular administration of the drugs, which could deeply affect the treatment outcome. To prevent this, we have implemented oral health management. In this study, the outcome of oral health management in the patients with malignant musculoskeletal tumor was retrospectively investigated.

\section{Methods}

Included: Patients receiving chemotherapy for malignant musculoskeletal tumor and professional oral health care from January 2012 to January 2019. Excluded: Edentulous patients / No examination of the periodontal pocket depth and panorama X-ray / Tumor or metastasis located in head and neck. Grading of tooth infection and neutropenia: CTCAE ver.5.0

\begin{tabular}{lll} 
※Tooth infection grade & G1 & G2 \\
\cline { 2 - 3 } & - & Localized; local intervention indicated (e.g., topical
\end{tabular} antibiotic, antifungal, or antiviral)
G3

IV antibiotic, antifungal, or antiviral intervention indicated; invasive intervention indicated

Results

Patient characteristics (n:71)

\begin{tabular}{|c|c|c|}
\hline \multicolumn{2}{|c|}{ All / Oral management / Include } & $106 / 102 / 71$ \\
\hline \multicolumn{2}{|c|}{ Sex: male / female } & $37 / 34$ \\
\hline \multicolumn{2}{|c|}{ Age (median) } & $43(11-76)$ \\
\hline \multicolumn{2}{|c|}{ Bone cancer / Soft tissue sarcoma } & $31 / 40$ \\
\hline \multirow{4}{*}{$\begin{array}{c}\text { Stage } \\
\text { (Bone cancer / } \\
\text { Soft tissue } \\
\text { sarcoma) }\end{array}$} & 2 & $14 / 4$ \\
\hline & 3 & $0 / 19$ \\
\hline & 4 & $9 / 11$ \\
\hline & recurrence or metastasis & $7 / 7$ \\
\hline \multicolumn{2}{|c|}{ PS: $0 / 1 / 2 / 3$} & $7 / 46 / 17 / 1$ \\
\hline \multicolumn{2}{|c|}{ Neutropenia G3 or G4 } & 63 \\
\hline
\end{tabular}

Dental characteristics ( $\mathrm{n}: 71)$

$\left(\begin{array}{c|c}\text { Number of remaining teeth (median) } & 28(2-32) \\ \hline \text { 3rd molar } & 49 \\ \hline \text { Semi-impacted or impacted 3rd molar } & 32 \\ \hline \geqq 6 m m \text { periodontal pocket } & 14 \\ \hline \text { Periapical lesion } & 33 \\ \hline \text { Removal of dental calculus } & 71 \\ \hline \text { Instruction for oral hygiene } & 71 \\ \hline \text { Dental treatment } & 20 \\ \hline \text { Tooth extraction } & 8\end{array}\right)$

\section{Tooth infection (n:10)}

\begin{tabular}{|c|c|c|c|c|c|c|c|c|}
\hline No & Malignant tumor & Chemotherapy & $\begin{array}{l}\text { Tooth } \\
\text { infection }\end{array}$ & Grade & Dental status & $\begin{array}{c}\text { Neutropenia } \\
\text { grade }\end{array}$ & $\mathrm{FN}$ & \\
\hline 1 & Osteosarcoma & $\begin{array}{c}\mathrm{MTX}+\mathrm{ADM}+ \\
\mathrm{CDDP}\end{array}$ & periodontitis & 2 & $\begin{array}{c}<6 \text { mm periodontal } \\
\text { pocket depth }\end{array}$ & 4 & No & \\
\hline 2 & $\begin{array}{l}\text { Dedifferentiated } \\
\text { liposarcoma }\end{array}$ & $\mathrm{IFO}+\mathrm{ADM}$ & periodontitis & 2 & $\begin{array}{l}\geqq 6 \mathrm{~mm} \text { periodontal } \\
\text { pocket depth }\end{array}$ & 0 & No & 8 \\
\hline 3 & Osteosarcoma & ADM & pericoronitis & 2 & semi-impacted & 4 & No & \\
\hline 4 & Osteosarcoma & IFO + VP16 & $\begin{array}{c}\text { apical } \\
\text { periodontitis }\end{array}$ & 2 & $\begin{array}{c}<5 \text { mm periapical } \\
\text { lesion size }\end{array}$ & 2 & No & \\
\hline 5 & Rhabdomyosarcoma & $\begin{array}{c}\text { VCR + Act-D + } \\
\text { CPA }\end{array}$ & pericoronitis & 3 & semi-impacted & 4 & Yes & \\
\hline 6 & Soft tissue sarcoma & GEM + DOC & periodontitis & 2 & $\begin{array}{c}\geqq 6 \mathrm{~mm} \text { periodontal } \\
\text { pocket depth }\end{array}$ & NA & No & \\
\hline 7 & Synovial sarcoma & $\mathrm{IFO}+\mathrm{ADM}$ & pericoronitis & 2 & not-impacted & 3 & No & \\
\hline 8 & $\begin{array}{c}\text { Malignant peripheral } \\
\text { nerve sheath tumor }\end{array}$ & $\mathrm{IFO}+\mathrm{ADM}$ & pericoronitis & 2 & not-impacted & 3 & No & \\
\hline 9 & Osteosarcoma & IFO + VP16 & pericoronitis & - & impacted & 0 & No & \\
\hline 10 & Synovial sarcoma & $\mathrm{IFO}+\mathrm{ADM}$ & pericoronitis & - & not-impacted & 3 & No & \\
\hline
\end{tabular}

Discussion

Pericoronitis frequently occurred. Especially, in cases of semi-impacted or impacted $3^{\text {rd }}$ molar, the extraction was invasive, and it may not have been in time for chemotherapy. Thus, these teeth tended to be left. The patients had other teeth with problems as well, so it was difficult to decide which tooth to treat. There may be other factors aside from radiographic image and periodontal pocket depth that predict tooth infection, such as poor oral hygiene.

\section{Conclusions}

Oral health management during chemotherapy for malignant musculoskeletal tumor was important. More studies are required to prevent febrile neutropenia from tooth infection. 